\title{
The Laplacian polynomial and Kirchhoff index of graphs based on $R$-graphs
}

\author{
Qun $\mathrm{Liu}^{\mathrm{a}}$, Jia-Bao Liu ${ }^{\mathrm{b}}$, Jinde $\mathrm{CaO}^{\mathrm{c}, \mathrm{d}, *}$ \\ ${ }^{a}$ Department of Mathematics and Statistics, Lanzhou University, Lanzhou 730000, China \\ ${ }^{b}$ Department of Public Courses, Anhui Xinhua University, Hefei 230088, China \\ ${ }^{c}$ Research Center for Complex Systems and Network Science, Department of Mathematics, Southeast University, Nanjing 210096, \\ China \\ ${ }^{d}$ Department of Mathematics, Faculty of Science, King Abdulazez University, Jeddah 21589, Saudi Arabia
}

\begin{abstract}
Let $R(G)$ be the graph obtained from $G$ by adding a new vertex corresponding to each edge of $G$ and by joining each new vertex to the end vertices of the corresponding edge. Let $I(G)$ be the set of newly added vertices. The $R$-vertex corona of $G_{1}$ and $G_{2}$, denoted by $G_{1} \odot G_{2}$, is the graph obtained from vertex disjoint $R\left(G_{1}\right)$ and $\left|V\left(G_{1}\right)\right|$ copies of $G_{2}$ by joining the ith vertex of $V\left(G_{1}\right)$ to every vertex in the ith copy of $G_{2}$. The $R$-edge corona of $G_{1}$ and $G_{2}$, denoted by $G_{1} \ominus G_{2}$, is the graph obtained from vertex disjoint $R\left(G_{1}\right)$ and $\left|I\left(G_{1}\right)\right|$ copies of $G_{2}$ by joining the ith vertex of $I\left(G_{1}\right)$ to every vertex in the ith copy of $G_{2}$. Liu et al. gave formulae for the Laplacian polynomial and Kirchhoff index of $R T(G)$ in [19]. In this paper, we give the Laplacian polynomials of $G_{1} \odot G_{2}$ and $G_{1} \ominus G_{2}$ for a regular graph $G_{1}$ and an arbitrary graph $G_{2}$; on the other hand, we derive formulae and lower bounds of Kirchhoff index of these graphs and generalize the existing results.
\end{abstract}

(C) 2011 Published by Elsevier Ltd.

Keywords: Kirchhoff index, Resistance distance, Corona, Edge corona, Laplacian polynomial, Schur complement

\section{Introduction}

Throughout this article, all graphs considered are simple and undirected. Let $G=(V(G), E(G))$ be a graph with vertex set $V(G)=\left\{v_{1}, v_{2}, \ldots, v_{n}\right\}$ and edge set $E(G)=\left\{e_{1}, e_{2}, \ldots, e_{m}\right\}$. The adjacency matrix of $G$, denoted by $A(G)$, is an $n \times n$ symmetric matrix such that $a_{i j}=1$ if vertices $v_{i}$ and $v_{j}$ are adjacent and 0 otherwise. Let $d_{i}=d_{G}\left(v_{i}\right)$ be the degree of vertex $v_{i}$ in $G$ and $D(G)=\operatorname{diag}\left(d_{1}, d_{2}, \ldots, d_{n}\right)$ be the diagonal matrix of vertex degrees. The Laplacian matrix of $G$ are defined as $L(G)=D(G)-A(G)$. Denoted by $P_{G}(x)$ and $\mu_{G}(x)$ the adjacent characteristic polynomial $\operatorname{det}(x I-A(G))$ and the Laplacian characteristic polynomial $\operatorname{det}(x I-L(G))$ of $G$, respectively. Since $A(G)$ and $L(G)$ are all real symmetric matrices, their eigenvalues are real numbers. So we can assume that $\lambda_{1}(G) \geq \lambda_{2}(G) \geq \ldots \geq \lambda_{n}(G)$ (resp., $\left.0=\mu_{1}(G) \leq \mu_{2}(G) \leq \ldots \leq \mu_{n}(G)\right)$ are the adjacency(resp., Laplacian ) eigenvalues of $G$. The collection of the adjacency(resp., Laplacian ) eigenvalues of $G$ together with their multiplicities forms the adjacency(resp., Laplacian) spectrum of $G$. For other undefined notations and terminology from graph theory, the readers may refer to [1] and the

\footnotetext{
${ }^{*}$ Corresponding author

Email addresses: liuqun09@yeah.net (Qun Liu), liujiabaoad@163.com (Jia-Bao Liu), jdcao@seu.edu.cn (Jinde Cao)
} 
references therein. There are many applications for Lapalacian eigenvalues of graphs. For example, there are many problems in physics and chemistry where the Laplacian eigenvalues play the central role. The Laplacian eigenvalues are in the segmentation of the combination optimization, method of design, parallel algorithm, solving linear systems, clustering and other aspects of a wide range of applications. See [33],[34].

In 1993, Klein and Randić [2] introduced a distance function named resistance distance on the basis of electrical network theory. They view a graph as an electrical network each edge of the graph is assumed to be a unit resistor, then take the resistance distance between vertices to be the effective resistance between them. Let $G$ be a simple graph with the vertex set $V(G)=\left\{v_{1}, v_{2}, \ldots, v_{n}\right\}$, and $r_{i j}$ denote the effective resistance distance between vertices $v_{i}$ and $v_{j}$ as computed with Ohm's law when all the edges of $G$ are considered to be unit resistors. The sum of resistance distance $K f(G)=\sum_{i<j} r_{i j}(G)$ was proposed in [1], later called the Kirchhoff index of $G$ in [3]. In electric theory, it is of interest to compute the effective resistance between any pair of vertices of a network, as well as the Kirchhoff index. The Kirchhoff index was introduced in chemistry as a better alternative to other parameters used for discriminating different molecules with similar shapes and structures. See [2]. The resistance distance and the Kirchhoff index attracted extensive attention due to its wide applications in physics, chemistry, etc. See [4]-[9]). For more information on resistance distance and Kirchhoff index of graphs, the readers are referred to the papers ([7]-[9]).

In [10], new graph operations based on $R(G)$ graphs: $R$-vertex corona and $R$-edge corona, are introduced, and their $A$-spectrum(resp., $L$-spectrum) are investigated. For a graph $G$, Let $R(G)$ be the graph obtained from $G$ by adding a new vertex $u_{e}$ and joining $u_{e}$ to the end vertices of $e$ for each $e \in E(G)$. The graph $R(G)$ appeared in [11] and we call it the $R$ - graph of $G$. Let $I(G)$ be the set of newly added vertices, i.e $I(G)=V(R(G)) \backslash V(G)$.

Let $G_{1}$ and $G_{2}$ be two vertex-disjoint graphs.

Definition 1.1 ([10]) The $R$-vertex corona of $G_{1}$ and $G_{2}$, denoted by $G_{1} \odot G_{2}$, is the graph obtained from vertex disjoint $R\left(G_{1}\right)$ and $\left|V\left(G_{1}\right)\right|$ copies of $G_{2}$ by joining the ith vertex of $V\left(G_{1}\right)$ to every vertex in the ith copy of $G_{2}$.

Definition 1.2 ([10]) The $R$-edge corona of $G_{1}$ and $G_{2}$, denoted by $G_{1} \ominus G_{2}$, is the graph obtained from vertex disjoint $R\left(G_{1}\right)$ and $\left|I\left(G_{1}\right)\right|$ copies of $G_{2}$ by joining the ith vertex of $\left|I\left(G_{1}\right)\right|$ to every vertex in the ith copy of $G_{2}$.

Note that if $G_{i}$ has $n_{i}$ vertices and $m_{i}$ edges for $i=1,2$, then $G_{1} \odot G_{2}$ has $n_{1}+m_{1}+n_{1} n_{2}$ vertices and $3 m_{1}+n_{1} m_{2}+n_{1} n_{2}$ edges, $G_{1} \ominus G_{2}$ has $n_{1}+m_{1}+m_{1} n_{2}$ vertices and $3 m_{1}+m_{1} m_{2}+m_{1} n_{2}$ edges.

As the authors of [12] pointed out, it is an interesting problem to compute Kirchhoff index of large composition graphs in terms of parameters of small graph in the composition $[13,14]$. The Kirchhoff index has been computed for some classes of graphs, such as cycles [15], complete graph [15], distance transitive graphs [16], and so on $[5,8,15,17,20,21,22,23,24,25,32]$. The Kirchhoff index of certain composite operations between two graphs was studied as well, such as product, lexicographic product [18] and join, corona, cluster [12]. Then recently Liu et al. [19] explore the Laplacian polynomial of $R T(G)$ of a regular graph $G$. Motivated by these results, in this paper we compute the Laplacian polynomial of $G_{1} \odot G_{2}$ and $G_{1} \ominus G_{2}$ for a regular graph $G_{1}$ and an arbitrary graph $G_{2}$ and derive formulae and low bounds of Kirchhoff index of these graphs and generalize their results in [19].

\section{Preliminaries}

In this section, we determine the characteristic polynomials of graphs with the help of the coronal of a matrix. The $M$-coronal $T_{M}(\lambda)$ of an $n \times n$ matrix $M$ is defined [26, 27] to be the sum of the entries of the matrix $\left(\lambda I_{n}-M\right)^{-1}$, that is

$$
T_{M}(\lambda)=\mathbf{1}_{\mathbf{n}}^{\mathbf{T}}\left(\lambda \mathbf{I}_{\mathbf{n}}-\mathbf{M}\right)^{-\mathbf{1}} \mathbf{1}_{\mathbf{n}},
$$

where $\mathbf{1}_{n}$ denotes the column vector of dimension $n$ with all the entries equal one.

If $M$ has a constant row sum $t$, it is easy to verify that

$$
T_{M}(\lambda)=\frac{n}{\lambda-t} .
$$

The Kronecker product $A \otimes B$ of two matrices $A=\left(a_{i j}\right)_{m \times n}$ and $B=\left(b_{i j}\right)_{p \times q}$ is the $m p \times n q$ matrix obtained from $A$ by replacing each element $a_{i j}$ by $a_{i j} B$. This is an associate operation with the property that $(A \otimes B)^{T}=A^{T} \otimes B^{T}$ and $(A \otimes B)(C \otimes D)=A C \otimes B D$ whenever the products $A C$ and $B D$ exists. 
Lemma 2.1 [29] Let $M_{1}, M_{2}, M_{3}$ and $M_{4}$ be respectively $p \times p, p \times q, q \times p$ and $q \times q$ matrices with $M_{1}$ and $M_{4}$ invertible, then

$$
\begin{aligned}
\operatorname{det}\left(\begin{array}{ll}
M_{1} & M_{2} \\
M_{3} & M_{4}
\end{array}\right) & =\operatorname{det}\left(M_{4}\right) \operatorname{det}\left(M_{1}-M_{2} M_{4}^{-1} M_{3}\right) \\
& =\operatorname{det}\left(M_{1}\right) \operatorname{det}\left(M_{4}-M_{3} M_{4}^{-1} M_{2}\right),
\end{aligned}
$$

where $M_{1}-M_{2} M_{4}^{-1} M_{3}$ and $M_{4}-M_{3} M_{1}^{-1} M_{2}$ are called the Schur complements of $M_{4}$ and $M_{1}$, respectively.

\section{The Laplacian polynomial of $R$-vertex and $R$-edge corona}

For a regular graph $G_{1}$, the next theorems give the representation of the Laplacian polynomial of $G_{1} \odot G_{2}$ and $G_{1} \ominus G_{2}$ by means of the characteristic polynomial and the Laplacian polynomial of $G_{1}$ and $G_{2}$.

Theorem 3.1 Let $G_{1}$ be an $r_{1}$-regular graph with $n_{1}$ vertices and $m_{1}$ edges and $G_{2}$ be an arbitrary graph with $n_{2}$ vertices. Then the Laplacian characteristic polynomial of $G_{1} \odot G_{2}$ is given by

(i) $\mu_{G_{1} \odot G_{2}}(x)=\prod_{i=1}^{n_{2}}\left(x-1-\mu_{i}\left(G_{2}\right)\right)^{n_{1}}(x-2)^{m_{1}}(3-x)^{n_{1}} P_{G_{1}}\left(\frac{\left(x-n_{2}\right)(x-2)}{3-x}+\frac{r_{1}(2 x-3)}{x-3}+\frac{n_{2}(x-2)}{(x-3)(x-1)}\right)$.

(ii) $\mu_{G_{1} \odot G_{2}}(x)=\prod_{i=1}^{n_{2}}\left(x-1-\mu_{i}\left(G_{2}\right)\right)^{n_{1}}(x-2)^{m_{1}}(x-3)^{n_{1}} \mu_{G_{1}}\left(\frac{x\left(x^{2}-\left(3+n_{2}+r_{1}\right) x+\left(2 n_{2}+r_{1}+2\right)\right)}{(x-1)(x-3)}\right)$.

Proof (i) Let $B$ be the vertex-edge incidence matrix of $G_{1}$. Since $G_{1}$ is an $r_{1}$ - regular graph, we have $D\left(G_{1}\right)=r_{1} I_{n_{1}}$. By a pertinent labeling of the vertices of $G_{1} \odot G_{2}$, then the Laplacian matrix of $G_{1} \odot G_{2}$ can be written as

$$
L\left(G_{1} \odot G_{2}\right)=\left(\begin{array}{ccc}
2 I_{m_{1}} & -B^{T} & 0_{m_{1} \times n_{1} n_{2}} \\
-B & \left(r_{1}+n_{2}\right) I_{n_{1}}+L\left(G_{1}\right) & -I_{n_{1}} \otimes 1_{n_{2}}^{T} \\
0_{n_{1} n_{2} \times m_{1}} & -I_{n_{1}} \otimes 1_{n_{2}} & I_{n_{1}} \otimes\left(I_{n_{2}}+L\left(G_{2}\right)\right)
\end{array}\right),
$$

where $0_{n}$ denotes the length- $n$ column vectors consisting entirely of $0^{\prime} s$.

It follows that

$$
\begin{gathered}
\mu_{G_{1} \odot G_{2}}(x)=\operatorname{det}\left(\begin{array}{ccc}
(x-2) I_{m_{1}} & B^{T} & 0_{m_{1} \times n_{1} n_{2}} \\
B & \left(x-r_{1}-n_{2}\right) I_{n_{1}}-L\left(G_{1}\right) & I_{n_{1}} \otimes 1_{n_{2}}^{T} \\
0_{n_{1} n_{2} \times m_{1}} & I_{n_{1}} \otimes 1_{n_{2}} & I_{n_{1}} \otimes\left((x-1) I_{n_{2}}-L\left(G_{2}\right)\right)
\end{array}\right) \\
=\prod_{i=1}^{n_{2}}\left(x-1-\mu_{i}\left(G_{2}\right)\right)^{n_{1}} \cdot \operatorname{det}(S),
\end{gathered}
$$

where

$$
\begin{aligned}
& S=\left(\begin{array}{cc}
(x-2) I_{m_{1}} & B^{T} \\
B & \left(x-r_{1}-n_{2}\right) I_{n_{1}}-L\left(G_{1}\right)
\end{array}\right)-\left(\begin{array}{c}
0_{m_{1} \times n_{1} n_{2}} \\
I_{n_{1}} \otimes 1_{n_{2}}^{T}
\end{array}\right) \\
& \left(I_{n_{1}} \otimes\left((x-1) I_{n_{2}}-L\left(G_{2}\right)\right)^{-1}\left(\begin{array}{ll}
0_{n_{1} n_{2} \times m_{1}} & I_{n_{1}} \otimes 1_{n_{2}}
\end{array}\right)\right. \\
& =\left(\begin{array}{cc}
(x-2) I_{m_{1}} & B^{T} \\
B & \left(x-r_{1}-n_{2}\right) I_{n_{1}}-L\left(G_{1}\right)
\end{array}\right)-\left(\begin{array}{cc}
0_{m_{1} \times m_{1}} & 0_{m_{1} \times n_{1}} \\
0_{n_{1} \times m_{1}} & I_{n_{1}} \otimes 1_{n_{2}}^{T}\left(x I_{n_{2}}-L\left(G_{2}\right)\right)^{-1} 1_{n_{2}}
\end{array}\right) \\
& =\left(\begin{array}{cc}
(x-2) I_{m_{1}} & B^{T} \\
B & \left(x-r_{1}-n_{2}\right) I_{n_{1}}-L\left(G_{1}\right)
\end{array}\right)-\left(\begin{array}{cc}
0_{m_{1} \times m_{1}} & 0_{m_{1} \times n_{1}} \\
0_{n_{1} \times m_{1}} & T_{L\left(G_{2}\right)}(x) I_{n_{1}}
\end{array}\right) \\
& =\left(\begin{array}{cc}
(x-2) I_{m_{1}} & B^{T} \\
B & \left(x-r_{1}-n_{2}-T_{L\left(G_{2}\right)}(x)\right) I_{n_{1}}-L\left(G_{1}\right)
\end{array}\right) .
\end{aligned}
$$

From (1), we have $T_{L\left(G_{2}\right)}(x-1)=\frac{n_{2}}{x-1}$ as each row sum of $L\left(G_{2}\right)$ is equal to 0 . It is well known that $B B^{T}=A\left(G_{1}\right)+r_{1} I_{n_{1}}$. Consequently,

$$
\begin{aligned}
\operatorname{det}(S) & =\operatorname{det}\left((x-2) I_{m_{1}}\right) \operatorname{det}\left(\left(x-r_{1}-n_{2}-\frac{n_{2}}{x-1}\right) I_{n_{1}}-L\left(G_{1}\right)-\frac{1}{x-2} B B^{T}\right) \\
& =(x-2)^{m_{1}} \operatorname{det}\left(\left(x-2 r_{1}-n_{2}-\frac{n_{2}}{x-1}-\frac{r_{1}}{x-2}\right) I_{n_{1}}+\left(1-\frac{1}{x-2}\right) A\left(G_{1}\right)\right) \\
& =(x-2)^{m_{1}}(3-x)^{n_{1}} \operatorname{det}\left(\left(\frac{\left(x-n_{2}\right)(x-2)}{3-x}+\frac{r_{1}(2 x-3)}{x-3}+\frac{n_{2}(x-2)}{(x-3)(x-1)}\right) I_{n_{1}}-A\left(G_{1}\right)\right)
\end{aligned}
$$




$$
=(x-2)^{m_{1}}(3-x)^{n_{1}} P_{G_{1}}\left(\frac{\left(x-n_{2}\right)(x-2)}{3-x}+\frac{r_{1}(2 x-3)}{x-3}+\frac{n_{2}(x-2)}{(x-3)(x-1)}\right) .
$$

By virtue of (2) and (3) we have already established the statement (i) in Theorem 3.1.

(ii) Recall that $L(G)=r I_{n}-A(G)$. It follows from that

$$
\begin{aligned}
\operatorname{det}(S) & =(x-2)^{m_{1}-n_{1}} \operatorname{det}\left(\left(x-2 r_{1}-n_{2}-\frac{n_{2}}{x-1}-\frac{r_{1}}{x-2}\right) I_{n_{1}}+\frac{x-3}{x-2} A\left(G_{1}\right)\right) \\
& =(x-2)^{m_{1}-n_{1}}(x-3)^{n_{1}} \operatorname{det}\left(\left(\frac{\left(x-n_{2}\right)(x-2)}{x-3}-\frac{r_{1} x}{x-3}-\frac{n_{2}(x-2)}{(x-3)(x-1)}\right) I_{n_{1}}-\left(r_{1} I_{n_{1}}-A\left(G_{1}\right)\right)\right. \\
& =(x-2)^{m_{1}-n_{1}}(x-3)^{n_{1}} \mu_{G_{1}}\left(\frac{x\left(x^{2}-\left(3+n_{2}+r_{1}\right) x\left(2 n_{2}+r_{1}+2\right)\right)}{(x-1)(x-3)}\right) .
\end{aligned}
$$

The proof is thus completed.

Remark 3.2: In [19], the authors gave the Laplacian polynomial of $G_{1} \odot G_{2}$ when $G_{2}$ is $K_{2}$, but we obtain the Laplacian polynomial of $G_{1} \odot G_{2}$ when $G_{2}$ is an arbitrary graph, so we generalize Theorem 3.1 in [19].

Next, we consider the case for $G_{1} \ominus G_{2}$. For a regular graph, the next theorem gives the representation of the Laplacian polynomial of $G_{1} \ominus G_{2}$ by means of the characteristic polynomial and the Laplacian polynomial of $G_{1}$ and $G_{2}$.

Theorem 3.3 Let $G_{1}$ be an $r_{1}$ - regular graph with $n_{1}$ vertices and $m_{1}$ edges and $G_{2}$ be an arbitrary graph with $n_{2}$ vertices. Then the Laplacian characteristic polynomial of $G_{1} \ominus G_{2}$ is given by

(i) $\mu_{G_{1} \ominus G_{2}}(x)=\prod_{i=2}^{n_{2}}\left(x-1-\mu_{i}\left(G_{2}\right)\right)^{m_{1}}(-1)^{n_{1}}\left(x^{2}-\left(3+n_{2}\right) x+2\right)^{m_{1}-n_{1}}\left(x^{2}-\left(4+n_{2}\right) x+3\right)^{n_{1}} P_{G_{1}}\left(\frac{-x^{3}+\left(n_{2}+3+2 r_{1}\right) x-\left(2+2 r_{1} n_{2}+5 r_{1}\right) x+3 r_{1}}{x^{2}-\left(4+n_{2}\right) x+3}\right)$.

(ii) $\mu_{G_{1} \ominus G_{2}}(x)=\prod_{i=2}^{n_{2}}\left(x-1-\mu_{i}\left(G_{2}\right)\right)^{m_{1}}\left(x^{2}-\left(3+n_{2}\right) x+2\right)^{m_{1}-n_{1}}\left(x^{2}-\left(4+n_{2}\right) x+3\right)^{n_{1}} \mu_{G_{1}}\left(\frac{x\left[x^{2}-\left(3+n_{2}+r_{1}\right) x\left(r_{2} n_{2}+r_{1}+2\right)\right]}{(x-1)(x-3)}\right)$.

Proof (i) Let $B$ be the vertex-edge incidence matrix of $G_{1}$. Since $G_{1}$ is an $r_{1}$ - regular graph, we have $D\left(G_{1}\right)=r_{1} I_{n_{1}}$. By a pertinent labeling of the vertices of $G_{1} \ominus G_{2}$, then the Laplacian matrix of $G_{1} \ominus G_{2}$ can be written as

$$
L\left(G_{1} \ominus G_{2}\right)=\left(\begin{array}{ccc}
\left(n_{2}+2\right) I_{m_{1}} & -B^{T} & -I_{m_{1}} \otimes 1_{n_{2}}^{T} \\
-B & r_{1} I_{n_{1}}+L\left(G_{1}\right) & 0_{n_{1} \times m_{1} n_{2}} \\
-I_{m_{1}} \otimes 1_{n_{2}} & 0_{m_{1} n_{2} \times n_{1}} & I_{m_{1}} \otimes\left(I_{n_{2}}+L\left(G_{2}\right)\right)
\end{array}\right) .
$$

It follows that

$$
\begin{gathered}
\mu_{G_{1} \ominus G_{2}}(x)=\operatorname{det}\left(\begin{array}{ccc}
\left(x-n_{2}-2\right) I_{m_{1}} & B^{T} & I_{m_{1}} \otimes 1_{n_{2}}^{T} \\
B & \left(x-r_{1}\right) I_{n_{1}}-L\left(G_{1}\right) & 0_{n_{1} \times m_{1} n_{2}} \\
I_{m_{1}} \otimes 1_{n_{2}} & 0_{m_{1} n_{2} \times n_{1}} & I_{m_{1}} \otimes\left((x-1) I_{n_{2}}-L\left(G_{2}\right)\right)
\end{array}\right) \\
=\prod_{i=1}^{n_{2}}\left(x-1-\mu_{i}\left(G_{2}\right)\right)^{m_{1}} \cdot \operatorname{det}(S),
\end{gathered}
$$

where

$$
\begin{aligned}
& S=\left(\begin{array}{cc}
\left(x-n_{2}-2\right) I_{m_{1}} & B^{T} \\
B & \left(x-r_{1}\right) I_{n_{1}}-L\left(G_{1}\right)
\end{array}\right)-\left(\begin{array}{c}
I_{m_{1}} \otimes 1_{n_{2}}^{T} \\
0_{n_{1} \times m_{1} n_{2}}
\end{array}\right) \\
& \left(I _ { m _ { 1 } } \otimes ( ( x - 1 ) I _ { n _ { 2 } } - L ( G _ { 2 } ) ) ^ { - 1 } \left(\begin{array}{ll}
I_{m_{1}} \otimes 1_{n_{2}} & \left.0_{m_{1} n_{2} \times n_{1}}\right)
\end{array}\right.\right. \\
& =\left(\begin{array}{cc}
\left(x-n_{2}-2\right) I_{m_{1}} & B^{T} \\
B & \left(x-r_{1}\right) I_{n_{1}}-L\left(G_{1}\right)
\end{array}\right)-\left(\begin{array}{cc}
I_{m_{1}} \otimes 1_{n_{2}}^{T}\left(x I_{n_{2}}-L\left(G_{2}\right)\right)^{-1} 1_{n_{2}} & 0_{m_{1} \times n_{1}} \\
0_{n_{1} \times m_{1}} & 0_{n_{1} \times n_{1}}
\end{array}\right) \\
& =\left(\begin{array}{cc}
\left(x-n_{2}-2-T_{L\left(G_{2}\right)}(x-1)\right) I_{m_{1}} & B^{T} \\
B & \left(x-r_{1}\right) I_{n_{1}}-L\left(G_{1}\right)
\end{array}\right) .
\end{aligned}
$$

From (1), we have $T_{L\left(G_{2}\right)}(x-1)=\frac{n_{2}}{x-1}$ as each row sum of $L\left(G_{2}\right)$ is equal to 0 . Note that $B B^{T}=A\left(G_{1}\right)+r_{1} I_{n_{1}}$. Consequently,

$$
\begin{aligned}
\operatorname{det}(S)= & \operatorname{det}\left(\left(x-n_{2}-2-T_{L\left(G_{2}\right)}(x-1)\right) I_{m_{1}}\right) \operatorname{det}\left(\left(x-r_{1}\right) I_{n_{1}}-L\left(G_{1}\right)-\frac{B B^{T}}{x-n_{2}-2-T_{L\left(G_{2}\right)}(x-1)}\right) \\
= & \left(x-n_{2}-2-T_{L\left(G_{2}\right)}(x-1)\right)^{m_{1}} \operatorname{det}\left(\left(x-2 r_{1}-\frac{r_{1}}{x-n_{2}-2-T_{L\left(G_{2}\right)}(x-1)}\right) I_{n_{1}}\right. \\
& \left.+\left(1-\frac{1}{x-n_{2}-2-T_{L\left(G_{2}\right)}(x-1)}\right) A\left(G_{1}\right)\right) \\
= & (-1)^{n_{1}}\left(x^{2}-\left(3+n_{2}\right) x+2\right)^{m_{1}-n_{1}}\left(x^{2}-\left(4+n_{2}\right) x+3\right)^{n_{1}} P_{G_{1}}\left(\frac{r_{1}-\left(x-2 r_{1}\right)\left(x-n_{2}-2-T_{L\left(G_{2}\right)}(x-1)\right)}{x-n_{2}-3-T_{L\left(G_{2}\right)}(x-1)}\right)
\end{aligned}
$$




$$
=(-1)^{n_{1}}\left(x^{2}-\left(3+n_{2}\right) x+2\right)^{m_{1}-n_{1}}\left(x^{2}-\left(4+n_{2}\right) x+3\right)^{n_{1}} P_{G_{1}}\left(\frac{-x^{3}+\left(n_{2}+3+2 r_{1}\right) x-\left(2+2 r_{1} n_{2}+5 r_{1}\right) x+3 r_{1}}{x^{2}-\left(4+n_{2}\right) x+3}\right) .
$$

By virtue of (4) and (5) we have already established the statement (i) in Theorem 3.2.

(ii) Recall that $L(G)=r I_{n}-A(G)$. It follows from that

$$
\begin{aligned}
\operatorname{det}(S)= & \left(x-n_{2}-2-T_{L\left(G_{2}\right)}(x-1)\right)^{m_{1}} \operatorname{det}\left(\left(x-2 r_{1}-\frac{r_{1}}{x-n_{2}-2-T_{L\left(G_{2}\right)}(x-1)} I_{n_{1}}+\left(1-\frac{1}{x-n_{2}-2-T_{L\left(G_{2}\right)}(x-1)}\right) A\left(G_{1}\right)\right)\right. \\
= & \left(x^{2}-\left(3+n_{2}\right) x+2\right)^{m_{1}-n_{1}}\left(x^{2}-\left(4+n_{2}\right) x+3\right)^{n_{1}} \\
& \operatorname{det}\left(\left(\frac{\left(x-2 r_{1}\right)\left(x-n_{2}-2-T_{L\left(G_{2}\right)}(x-1)\right)-r_{1}}{x-n_{2}-3-T_{L\left(G_{2}\right)}(x-1)}+r_{1}\right) I_{n_{1}}-\left(r_{1} I_{n_{1}}-A\left(G_{1}\right)\right)\right. \\
= & \left.\left(x^{2}-\left(3+n_{2}\right) x+2\right)^{m_{1}-n_{1}}\left(x^{2}-\left(4+n_{2}\right) x+3\right)^{n_{1}} \mu_{G_{1}}\left(\frac{x\left[x^{2}-\left(3+n_{2}+r_{1}\right) x+\left(r_{2} n_{2}+r_{1}+2\right)\right]}{x^{2}-\left(4+n_{2}\right) x+3}\right)\right) .
\end{aligned}
$$

The proof is thus completed.

\section{The Kirchhoff index of $\boldsymbol{R}$-vertex and $\boldsymbol{R}$-edge corona}

In this section, we will explore the Kirchhoff index of the $G_{1} \odot G_{2}$ and $G_{1} \ominus G_{2}$ for a regular graph $G_{1}$ and an arbitrary graph $G_{2}$.

Gutman and Mohar [30] and Zhu et al. [31] established the relationship between the Laplacian spectrum and Kirchhoff index as follows:

Lemma 4.1 [30] Let $G$ be a connected graph with $n \geq 2$ vertices. Then

$$
K f(G)=n \sum_{i=1}^{n-1} \frac{1}{\mu_{i}} .
$$

Denote by $\delta_{i}$ the degree of vertex $v_{i} \in V(G)$. Zhou and Trinajsti. [22] proved that

Lemma 4.2 [17] Let $G$ be a connected graph with $n \geq 2$ vertices. Then

$$
K f(G) \geq-1+(n-1) \sum_{v_{i} \in V(G)} \frac{1}{\delta_{i}}
$$

with equality attained if and only if $G=K_{n}$ or $G=K_{t, n_{-t}}$ for $1 \leq t \leq\left\lfloor\frac{n}{2}\right\rfloor$.

The following lemma established a nice relationship between the Laplacian polynomial and Kirchhoff index as follows:

Lemma 4.3 [15] Let $G$ be a connected graph with $n \geq 2$ vertices and $\mu_{G}(x)=x^{n}+a_{1} x^{n-1}+\ldots+a_{n-1} x$. Then

$$
\frac{K f(G)}{n}=-\frac{a_{n-2}}{a_{n-1}}\left(a_{n-2}=1 \text { whenever } n=2\right) .
$$

Let $K_{n}$ denote the complete graph with $n$ vertices. If $G=K_{1}$, we have nothing to discuss. So we assume that $G \neq K_{1}$ throughout this paper. The following Theorem 4.4 generalizes the Theorem 4.4 in [19] and we get the generalized results.

Theorem 4.4 Let $G_{1}$ be an $r_{1}$ - regular graph with $n_{1}$ vertices and $m_{1}$ edges and $G_{2}$ be an arbitrary graph with $n_{2}$ vertices. Then

$$
\begin{aligned}
K f\left(G_{1} \odot G_{2}\right)= & \frac{\left(2 n_{2}+r_{1}+2\right)^{2}}{6} K f\left(G_{1}\right)+\frac{n_{1}\left(3+n_{2}+r_{1}\right)}{2}+\frac{2 n_{1}\left(n_{1}-1\right)\left(2+r_{1}+2 n_{2}\right)}{3} \\
& +\frac{n_{1}^{2}\left(r_{1}-2\right)\left(r_{1}+2+2 n_{2}\right)}{8}+\frac{n_{1}^{2}\left(2+r_{1}+2 n_{2}\right)}{2} \sum_{i=2}^{n_{2}} \frac{1}{1+\mu_{i}\left(G_{2}\right)} .
\end{aligned}
$$


Proof Let $\mu_{G}(x)=x^{n}+a_{1} x^{n-1}+a_{2} x^{n-2}+\ldots+a_{n-1} x$. It follows from Theorem 3.1 (ii) that

$$
\begin{aligned}
\mu_{G_{1} \odot G_{2}}(x)= & \prod_{i=1}^{n_{2}}\left(x-1-\mu_{i}\left(G_{2}\right)\right)^{n_{1}}(x-2)^{m_{1}-n_{1}}(x-3)^{n_{1}} \\
& {\left[x^{n_{1}}\left(\frac{x^{2}-\left(3+n_{2}+r_{1}\right) x+\left(2 n_{2}+r_{1}+2\right)}{(x-1)(x-3)}\right)^{n_{1}}+\ldots\right.} \\
& +a_{n_{1}-2} x^{2}\left(\frac{x^{2}-\left(3+n_{2}+r_{1}\right) x+\left(2 n_{2}+r_{1}+2\right)}{(x-1)(x-3)}\right)^{2} \\
& \left.+a_{n_{1}-1} x\left(\frac{x^{2}-\left(3+n_{2}+r_{1}\right) x+\left(2 n_{2}+r_{1}+2\right)}{(x-1)(x-3)}\right)\right] \\
= & \prod_{i=2}^{n_{2}}\left(x-1-\mu_{i}\left(G_{2}\right)\right)^{n_{1}}(x-2)^{m_{1}-n_{1}}\left[x^{n_{1}}\left(x^{2}-\left(3+n_{2}+r_{1}\right) x+\left(2 n_{2}+r_{1}+2\right)\right)^{n_{1}}\right. \\
& +\ldots+a_{n_{1}-2} x^{2}(x-1)^{n_{1}-2}(x-3)^{n_{1}-2}\left(x^{2}-\left(3+n_{2}+r_{1}\right) x+\left(2 n_{2}+r_{1}+2\right)\right)^{2} \\
& \left.+a_{n_{1}-1} x(x-1)^{n_{1}-1}(x-3)^{n_{1}-1}\left(x^{2}-\left(3+n_{2}+r_{1}\right) x+\left(2 n_{2}+r_{1}+2\right)\right)\right],
\end{aligned}
$$

where $x \neq 1,3$. So the coefficient of $x^{2}$ in $\mu_{G_{1} \odot G_{2}}(x)$ is

$$
\begin{aligned}
& (-2)^{m_{1}-n_{1}} \prod_{i=2}^{n_{2}}\left(-\left(1+\mu_{i}\left(G_{2}\right)\right)^{n_{1}}\left[a_{n_{1}-2}\left(2 n_{2}+r_{1}+2\right)^{2}(-1)^{n_{1}-2}(-3)^{n_{1}-2}\right.\right. \\
& +a_{n_{1}-1}\left(-\left(3+n_{2}+r_{1}\right)\right)(-1)^{n_{1}-1}(-3)^{n_{1}-1} \\
& +a_{n_{1}-1}\left(2 n_{2}+r_{1}+2\right)\left(n_{1}-1\right)(-1)^{n_{1}-2}(-3)^{n_{1}-1} \\
& \left.+a_{n_{1}-1}\left(2 n_{2}+r_{1}+2\right)(-1)^{n_{1}-1}\left(n_{1}-1\right)(-3)^{n_{1}-2}\right] \\
& +\left(m_{1}-n_{1}\right)(-2)^{m_{1}-n_{1}-1} \prod_{i=2}^{n_{2}}\left(1+\mu_{i}\left(G_{2}\right)\right)^{n_{1}} a_{n_{1}-1}\left(2 n_{2}+r_{1}+2\right)(-1)^{n_{1}-1}(-3)^{n_{1}-1} \\
& +(-2)^{m_{1}-n_{1}} \sum_{j=2}^{n_{2}} n_{1}\left(-\left(1+\mu_{j}\left(G_{2}\right)\right)^{n_{1}-1} \prod_{i=2, i \neq j}^{n_{2}}\left(-\left(1+\mu_{i}\left(G_{2}\right)\right)^{n_{1}} a_{n_{1}-1}\right.\right. \\
& \left(2 n_{2}+r_{1}+2\right)(-1)^{n_{1}-1}(-3)^{n_{1}-1}
\end{aligned}
$$

and the coefficient of $x$ in $\mu_{G_{1} \odot G_{2}}(x)$ is

$$
(-2)^{m_{1}-n_{1}} \prod_{i=2}^{n_{2}}\left(-\left(1+\mu_{i}\left(G_{2}\right)\right)^{n_{1}}\left(2 n_{2}+r_{1}+2\right)(-1)^{n_{1}-1}(-3)^{n_{1}-1} a_{n_{1}-1} .\right.
$$

Note that $G_{1} \odot G_{2}$ has $n_{1}+m_{1}+n_{1} n_{2}$ vertices. It follows from Lemma 4.3, (6) and (7) that

$$
\begin{aligned}
\frac{K f\left(G_{1} \odot G_{2}\right)}{n_{1}+m_{1}+n_{1} n_{2}}= & -\frac{a_{n_{1}-2}}{a_{n_{1}-1}} \frac{2 n_{2}+r_{1}+2}{3}+\frac{3+n_{2}+r_{1}}{2 n_{2}+r_{1}+2}+\frac{4\left(n_{1}-1\right)}{3} \\
& +\frac{m_{1}+n_{1}}{2}+\sum_{i=2}^{n_{2}} \frac{n_{1}}{1+\mu_{i}\left(G_{2}\right)} .
\end{aligned}
$$

Substituting the result of Lemma 4.3 and $m_{1}=\frac{n_{1} r_{1}}{2}$ into the above equation, we have

$$
\begin{aligned}
& K f\left(G_{1} \odot G_{2}\right)= \frac{2 n_{2}+r_{1}+2}{3 n_{1}}\left(n_{1}+m_{1}+n_{1} n_{2}\right) K f\left(G_{1}\right)+\frac{3+n_{2}+r_{1}}{2 n_{2}+r_{1}+2}\left(n_{1}+m_{1}+n_{1} n_{2}\right) \\
&+\frac{4\left(n_{1}-1\right)}{3}\left(n_{1}+m_{1}+n_{1} n_{2}\right)+\frac{m_{1}-n_{1}}{2}\left(n_{1}+m_{1}+n_{1} n_{2}\right) \\
& 6
\end{aligned}
$$




$$
\begin{aligned}
+ & n_{1}\left(n_{1}+m_{1}+n_{1} n_{2}\right) \sum_{i=2}^{n_{2}} \frac{n_{1}}{1+\mu_{i}\left(G_{2}\right)} \\
= & \frac{\left(2 n_{2}+r_{1}+2\right)^{2}}{6} K f\left(G_{1}\right)+\frac{n_{1}\left(3+n_{2}+r_{1}\right)}{2}+\frac{2 n_{1}\left(n_{1}-1\right)\left(2+r_{1}+2 n_{2}\right)}{3} \\
& +\frac{n_{1}^{2}\left(r_{1}-2\right)\left(r_{1}+2+2 n_{2}\right)}{8}+\frac{n_{1}^{2}\left(2+r_{1}+2 n_{2}\right)}{2} \sum_{i=2}^{n_{2}} \frac{1}{1+\mu_{i}\left(G_{2}\right)}
\end{aligned}
$$

Summing up, we complete the proof.

Remark 4.5: Comparison to the Laplacian polynomial and its Kirchhoff index of $R T(G)$ in [19], our results of the graph $G_{1} \odot G_{2}$ has generality. It is clear that handling the problems of Laplacian polynomial and Kirchhoff index are more difficult and complex, but we deduce those with a simple approach.

In what follows, we give a lower bound for the Kirchhoff index of $G_{1} \odot G_{2}$.

Corollary 4.6 Let $G_{1}$ be an $r_{1}$ - regular graph with $n_{1}$ vertices and $m_{1}$ edges and $G_{2}$ be an arbitrary graph with $n_{2}$ vertices. Then

$$
\begin{array}{r}
K f\left(G_{1} \odot G_{2}\right) \geq \frac{n_{1}\left(n_{1}-1\right)\left(2 n_{2}+r_{1}+2\right)^{2}}{6 r_{1}}+\frac{n_{1}\left(3+n_{2}+r_{1}\right)}{2}+\frac{n_{1}^{2}\left(r_{1}-2\right)\left(r_{1}+2+2 n_{2}\right)}{8} \\
+\frac{\left(4 n_{1}^{2}-4 n_{1}-2 n_{2}-r_{1}-2\right)\left(2+r_{1}+2 n_{2}\right)}{6}+\frac{n_{1}^{2}\left(2+r_{1}+2 n_{2}\right)}{2} \sum_{i=2}^{n_{2}} \frac{1}{1+\mu_{i}\left(G_{2}\right)} .
\end{array}
$$

Proof It follows from Lemmma 4.2 and Theorem 4.4 that

$$
\begin{aligned}
K f\left(G_{1} \odot G_{2}\right) \geq & \frac{\left(2 n_{2}+r_{1}+2\right)^{2}}{6}\left(\frac{n_{1}\left(n_{1}-1\right)}{r_{1}}-1\right)+\frac{n_{1}\left(3+n_{2}+r_{1}\right)}{2}+\frac{2 n_{1}\left(n_{1}-1\right)\left(2+r_{1}+2 n_{2}\right)}{3} \\
& +\frac{n_{1}^{2}\left(r_{1}-2\right)\left(r_{1}+2+2 n_{2}\right)}{8}+\frac{n_{1}^{2}\left(2+r_{1}+2 n_{2}\right)}{2} \sum_{i=2}^{n_{2}} \frac{1}{1+\mu_{i}\left(G_{2}\right)} \\
= & \frac{n_{1}\left(n_{1}-1\right)\left(2 n_{2}+r_{1}+2\right)^{2}}{6 r_{1}}+\frac{n_{1}\left(3+n_{2}+r_{1}\right)}{2}+\frac{n_{1}^{2}\left(r_{1}-2\right)\left(r_{1}+2+2 n_{2}\right)}{8} \\
& +\frac{\left(4 n_{1}^{2}-4 n_{1}-2 n_{2}-r_{1}-2\right)\left(2+r_{1}+2 n_{2}\right)}{6}+\frac{n_{1}^{2}\left(2+r_{1}+2 n_{2}\right)}{2} \sum_{i=2}^{n_{2}} \frac{1}{1+\mu_{i}\left(G_{2}\right)} .
\end{aligned}
$$

The following result is proved in a way that is certainly similar in spirt to the proof of Theorem 4.4.

Theorem 4.7 Let $G_{1}$ be an $r_{1}$ - regular graph with $n_{1}$ vertices and $m_{1}$ edges and $G_{2}$ be an arbitrary graph with $n_{2}$ vertices. Then

$$
\begin{aligned}
K f\left(G_{1} \ominus G_{2}\right)= & \frac{\left(r_{1} n_{2}+r_{1}+2\right)^{2}}{6} K f\left(G_{1}\right)+\frac{n_{1}\left(3+n_{2}+r_{1}\right)}{2}+\frac{\left(n_{1}^{2}-n_{1}\right)\left(n_{2}+4\right)\left(2+r_{1}+r_{1} n_{2}\right)}{6} \\
& +\frac{n_{1}^{2}\left(r_{1}-2\right)\left(3+n_{1}\right)\left(r_{1}+2+r_{1} n_{2}\right)}{8}+\frac{n_{1}^{2} r_{1}\left(2+r_{1}+r_{1} n_{2}\right)}{4} \sum_{i=2}^{n_{2}} \frac{1}{1+\mu_{i}\left(G_{2}\right)} .
\end{aligned}
$$

Proof Let $\mu_{G}(x)=x^{n}+a_{1} x^{n-1}+a_{2} x^{n-2}+\ldots+a_{n-1} x$. It follows from Theorem 3.2 (ii) that

$$
\begin{aligned}
\mu_{G_{1} \ominus G_{2}}(x)= & \prod_{i=2}^{n_{2}}\left(x-1-\mu_{i}\left(G_{2}\right)\right)^{m_{1}}\left(x^{2}-\left(3+n_{2}\right) x+2\right)^{m_{1}-n_{1}}\left(x^{2}-\left(4+n_{2}\right) x+3\right)^{n_{1}} \\
& {\left[x^{n_{1}}\left(\frac{x^{2}-\left(3+n_{2}+r_{1}\right) x+\left(r_{1} n_{2}+r_{1}+2\right)}{x^{2}-\left(4+n_{2}\right) x+3}\right)^{n_{1}}+\ldots\right.} \\
& +a_{n_{1}-2} x^{2}\left(\frac{x^{2}-\left(3+n_{2}+r_{1}\right) x+\left(r_{1} n_{2}+r_{1}+2\right)}{x^{2}-\left(4+n_{2}\right) x+3}\right)^{2}
\end{aligned}
$$




$$
\begin{aligned}
& \left.+a_{n_{1}-1} x\left(\frac{x^{2}-\left(3+n_{2}+r_{1}\right) x+\left(r_{1} n_{2}+r_{1}+2\right)}{x^{2}-\left(4+n_{2}\right) x+3}\right)\right] \\
= & \prod_{i=2}^{n_{2}}\left(x-1-\mu_{i}\left(G_{2}\right)\right)^{m_{1}}\left(x^{2}-\left(3+n_{2}\right) x+2\right)^{m_{1}-n_{1}} \\
& {\left[x^{n_{1}}\left(x^{2}-\left(3+n_{2}+r_{1}\right) x+\left(r_{1} n_{2}+r_{1}+2\right)\right)^{n_{1}}+\ldots+a_{n_{1}-2} x^{2}\left(x^{2}-\left(3+n_{2}\right.\right.\right.} \\
& \left.\left.+r_{1}\right) x+\left(r_{1} n_{2}+r_{1}+2\right)\right)^{2}\left(x^{2}-\left(4+n_{2}\right) x+3\right)^{n_{1}-2}+a_{n_{1}-1} x\left(x^{2}-\left(3+n_{2}\right.\right. \\
& \left.\left.\left.+r_{1}\right) x+\left(r_{1} n_{2}+r_{1}+2\right)\right)\left(x^{2}-\left(4+n_{2}\right) x+3\right)^{n_{1}-1}\right],
\end{aligned}
$$

so the coefficient of $x^{2}$ in $\mu_{G_{1} \ominus G_{2}}(x)$ is

$$
\begin{aligned}
& 2^{m_{1}-n_{1}} \prod_{i=2}^{n_{2}}\left(-\left(1+\mu_{i}\left(G_{2}\right)\right)\right)^{m_{1}}\left[a_{n_{1}-2}\left(r_{1} n_{2}+r_{1}+2\right)^{2} 3^{n_{1}-2}\right. \\
& \quad+a_{n_{1}-1}\left(-\left(3+n_{2}+r_{1}\right)\right) 3^{n_{1}-1} \\
& \left.\quad+a_{n_{1}-1}\left(r_{1} n_{2}+r_{1}+2\right)\left(n_{1}-1\right)(-1)^{n_{1}-2} 3^{n_{1}-2}\left(-\left(4+n_{2}\right)\right)\left(n_{1}-1\right)\right]
\end{aligned}
$$

and the coefficient of $x$ in $\mu_{G_{1} \ominus G_{2}}(x)$ is

$$
2^{m_{1}-n_{1}} \prod_{i=2}^{n_{2}}\left(-\left(1+\mu_{i}\left(G_{2}\right)\right)^{m_{1}}\left(r_{1} n_{2}+r_{1}+2\right) 3^{n_{1}-1} a_{n_{1}-1} .\right.
$$

Note that $G_{1} \ominus G_{2}$ has $n_{1}+m_{1}+n_{1} n_{2}$ vertices. It follows that

$$
\begin{aligned}
\frac{K f\left(G_{1} \ominus G_{2}\right)}{n_{1}+m_{1}+m_{1} n_{2}}= & -\frac{a_{n_{1}-2}}{a_{n_{1}-1}} \frac{r_{1} n_{2}+r_{1}+2}{3}+\frac{3+n_{2}+r_{1}}{r_{1} n_{2}+r_{1}+2}+\frac{\left(n_{1}-1\right)\left(4+n_{2}\right)}{3} \\
& +\frac{\left(m_{1}-n_{1}\right)\left(3+n_{2}\right)}{2}+\sum_{i=2}^{n_{2}} \frac{m_{1}}{1+\mu_{i}\left(G_{2}\right)} .
\end{aligned}
$$

Substituting the result of Lemma 4.3 and $m_{1}=\frac{n_{1} r_{1}}{2}$ into the above equation, we have

$$
\begin{aligned}
K f\left(G_{1} \ominus G_{2}\right)= & \frac{\left(r_{1} n_{2}+r_{1}+2\right)^{2}}{6} K f\left(G_{1}\right)+\frac{n_{1}\left(3+n_{2}+r_{1}\right)}{2}+\frac{\left(n_{1}^{2}-n_{1}\right)\left(n_{2}+4\right)\left(2+r_{1}+r_{1} n_{2}\right)}{6} \\
& +\frac{n_{1}^{2}\left(r_{1}-2\right)\left(3+n_{1}\right)\left(r_{1}+2+r_{1} n_{2}\right)}{8}+\frac{n_{1}^{2} r_{1}\left(2+r_{1}+r_{1} n_{2}\right)}{4} \sum_{i=2}^{n_{2}} \frac{1}{1+\mu_{i}\left(G_{2}\right)} .
\end{aligned}
$$

Summing up, we complete the proof.

Corollary 4.8 Let $G_{1}$ be an $r_{1}$ - regular graph with $n_{1}$ vertices and $m_{1}$ edges and $G_{2}$ be an arbitrary graph with $n_{2}$ vertices. Then

$$
\begin{gathered}
K f\left(G_{1} \ominus G_{2}\right) \geq \frac{\left(n_{1}^{2}-n_{1}\right)\left(r_{1} n_{2}+r_{1}+2\right)^{2}}{6 r_{1}}+\frac{n_{1}\left(3+n_{2}+r_{1}\right)}{2}+\frac{\left(n_{1}^{2}-n_{1}\right)\left(n_{2}+4\right)\left(2+r_{1}+r_{1} n_{2}\right)}{6} \\
+\frac{\left(3 n_{1}^{2} r_{1}-2 r_{1} n_{2}-2 r_{1}-4\right)\left(2+r_{1}+r_{1} n_{2}\right)}{12}+\frac{n_{1}^{2}\left(r_{1}-2\right)\left(3+n_{1}\right)\left(r_{1}+2+r_{1} n_{2}\right)}{8} \sum_{i=2}^{n_{2}} \frac{1}{1+\mu_{i}\left(G_{2}\right)} .
\end{gathered}
$$


Proof It follows from Lemmma 4.2 and Theorem 4.6 that

$$
\begin{aligned}
K f\left(G_{1} \ominus G_{2}\right) \geq & \frac{\left(r_{1} n_{2}+r_{1}+2\right)^{2}}{6}\left(\frac{n_{1}\left(n_{1}-1\right)}{r_{1}}-1\right)+\frac{n_{1}\left(3+n_{2}+r_{1}\right)}{2}+\frac{n_{1}^{2} r_{1}\left(2+r_{1}+r_{1} n_{2}\right)}{4} \\
& +\frac{\left(n_{1}^{2}-n_{1}\right)\left(n_{2}+4\right)\left(2+r_{1}+r_{1} n_{2}\right)}{6}+\frac{n_{1}^{2}\left(r_{1}-2\right)\left(3+n_{1}\right)\left(r_{1}+2+r_{1} n_{2}\right)}{8} \sum_{i=2}^{n_{2}} \frac{1}{1+\mu_{i}\left(G_{2}\right)} \\
= & \frac{\left(n_{1}^{2}-n_{1}\right)\left(r_{1} n_{2}+r_{1}+2\right)^{2}}{6 r_{1}}+\frac{n_{1}\left(3+n_{2}+r_{1}\right)}{2}+\frac{\left(n_{1}^{2}-n_{1}\right)\left(n_{2}+4\right)\left(2+r_{1}+r_{1} n_{2}\right)}{6} \\
& +\frac{\left(3 n_{1}^{2} r_{1}-2 r_{1} n_{2}-2 r_{1}-4\right)\left(2+r_{1}+r_{1} n_{2}\right)}{12}+\frac{n_{1}^{2}\left(r_{1}-2\right)\left(3+n_{1}\right)\left(r_{1}+2+r_{1} n_{2}\right)}{8} \sum_{i=2}^{n_{2}} \frac{1}{1+\mu_{i}\left(G_{2}\right)} .
\end{aligned}
$$

At last, we give an example.

Example 4.9 Let $K_{n}$ a complete graph on $n$ vertices. Note that the eigenvalues of $L_{K_{n}}$ are 0 and $n$ repeated $n-1$ times. Then by Theorem 4.4 and Theorem 4.7, we have

$$
\begin{aligned}
K f\left(K_{n} \odot K_{n}\right)= & \frac{\left(2 n_{2}+r_{1}+2\right)^{2}}{6}\left(n_{1}-1\right)+\frac{n_{1}\left(3+n_{2}+r_{1}\right)}{2}+\frac{2 n_{1}\left(n_{1}-1\right)\left(2+r_{1}+2 n_{2}\right)}{3} \\
& +\frac{n_{1}^{2}\left(r_{1}-2\right)\left(r_{1}+2+2 n_{2}\right)}{8}+\frac{n_{1}^{2}\left(2+r_{1}+2 n_{2}\right)}{2}\left(\frac{n_{2}-1}{1+n_{2}}\right) . \\
K f\left(K_{n} \ominus K_{n}\right)= & \left.\frac{\left(r_{1} n_{2}+r_{1}+2\right)^{2}}{6}\left(n_{1}-1\right)\right)+\frac{n_{1}\left(3+n_{2}+r_{1}\right)}{2}+\frac{\left(n_{1}^{2}-n_{1}\right)\left(n_{2}+4\right)\left(2+r_{1}+r_{1} n_{2}\right)}{6} \\
& +\frac{n_{1}^{2}\left(r_{1}-2\right)\left(3+n_{1}\right)\left(r_{1}+2+r_{1} n_{2}\right)}{8}+\frac{n_{1}^{2} r_{1}\left(2+r_{1}+r_{1} n_{2}\right)}{4} \frac{n_{2}-1}{1+n_{2}} .
\end{aligned}
$$

\section{Conclusions}

In this paper, we explore the Laplacian polynomial of $G_{1} \odot G_{2}$ and $G_{1} \ominus G_{2}$ for a regular graph $G_{1}$ and an arbitrary graph $G_{2}$ and derive formulae for Kirchhoff index of these graphs. By utilizing the spectral graph theory, we establish the explicit formulas for $K f\left(G_{1} \odot G_{2}\right)$ and $K f\left(G_{1} \ominus G_{2}\right)$ in terms of $K f\left(G_{1}\right), K f\left(G_{2}\right)$, the number of vertices and the vertex degree of regular graph $G_{1}$ and $G_{2}$, based on which we propose a lower bound for the Kirchhoff index for $G_{1} \odot G_{2}$ and $G_{1} \ominus G_{2}$ with respect to the number of vertices and the vertex degree.

\section{Acknowledgments}

The authors are grateful to the anonymous referees for many valuable comments and suggestions, which led to great improvements of the original manuscript. This work was supported by the National Natural Science Foundation of China (no.11461020) and the Youth Foundation of Hexi University in Gansu Province (no.QN2013-07). The work of J.B. Liu was partly supported by the Natural Science Foundation for the Higher Education Institutions of Anhui Province of China under Grant no. KJ2015A331, and the National Science Foundation of China under Grant nos. 11471016 and 11401004 . The work of J. Cao was partly supported by the National Natural Science Foundation of China under Grant 61272530, the Natural Science Foundation of Jiangsu Province of China under Grant BK2012741, the Specialized Research Fund for the Doctoral Program of Higher Education under Grants 20110092110017 and 20130092110017. 


\section{References}

[1] D. Cvetković, M. Doob, H. Sachs, Spectra of Graphs-Theory and Application, Academic Press, New York, 1980.

[2] D. J. Klein, M. Randić, Resistance distance, J. Math. Chem. 12 (1993) 81-95.

[3] D. Bonchev, A.T. Balaban, X.Liu, D.J. Klein, Molecular cyclicity and centricity of polycyclic graphs. I: cyclicity based on resistance distances or reciprocal distances, Int. J. Quantum Chem. 50 (1994) 1-20.

[4] L. H. Feng, G. Yu, W. Liu, Further resuts regarding the degree Kirchhoff index of a graph, Miskolc Mathematical Notes. 151 (2014) 97-108.

[5] L. H. Feng, G. Yu, K. Xu, Z. Jiang, A note on the Kirchhoff index of bicyclic graphs, Ars Comb. 114 (2014) 33-40.

[6] L. H. Feng, Ivan Gutman, Guihai Yu, Degree Kirchhoff index of unicyclic graphs, MATCH Commun. Math. Comput. Chem. 69 (2013) 629-648.

[7] Y. J. Yang, D. J. Klein, Comparison theorems on resistance distances and Kirchhoff indices of S,T-isomers, Discrete Appl. Math. 175 (2014) 87-93.

[8] Y. J. Yang, The Kirchhoff index of subdivisions of graphs, Discrete Appl. Math. 171 (2014) 153-157.

[9] Z. Zhang, Some physical and chemical indices of clique-inserted lattices, Journal of Statistical Mechanics: Theory and Experiment. 10 (2013) $1-12$.

[10] J. Lan, B. Zhou, Spectra of graph operations based on R-graph, Linear and Multilinear Algebra. (2014)1-22.

[11] Cvetković DM, Doob M, Sachs H. Spectra of graphs, theory and application. 3rd ed. Heidelberg: Johann Ambrosius Barth; 1995

[12] H. Zhang, Y. Yang, C. Li, Kirchhoff index of composite graphs, Discrete Appl. Math. 157 (2009) 2918-2927.

[13] J. B. Liu, X. F. Pan, J. Cao, F. F. Hu, A note on 'some physical and chemical indices of clique-inserted lattices', Journal of Statistical Mechanics: Theory and Experiment. 6 (2014), P06006.

[14] J. B. Liu, X. F. Pan, Y. Wang, J. Cao, The Kirchhoff index of folded hypercubes and some variant networks, Mathematical Problems in Engineering, vol. 2014, Article ID 380874, 9 pages, 2014.

[15] I. Lukovits, S. Nikolić, N. Trinajstić, Resistance distance in regular graphs, Int. J. Quantum Chem. 71 (1999) $217-225$.

[16] J. L. Palacios, Closed form formulae for Kirchhoff index, Int. J. Quantum Chem. 81 (2001) 135-140.

[17] B. Zhou, N. Trinajstić, A note on Kirchhoff index, Chem. Phys. Lett. 455 (2008) 120-123.

[18] H. Xu, The Laplacian spectrum and Kirchhoff index of product and lexicographic product of graphs, J. Xiamen Univ. (Nat. Sci.). 42 (2003) 552-554 (in Chinese).

[19] J.B. Liu, X.F. Pan, F.T. Hu, The laplacian polynomial of graphs derived from regular graphs and applications, Ars Combinatoria, in press.

[20] J.B. Liu, X.F. Pan, L. Yu, D. Li, Complete characterization of bicyclic graphs with minimal Kirchhoff index, Discrete Appl. Math. In press. http://dx.doi.org/10.1016/j.dam.2015.07.001.

[21] J.B. Liu, X.F. Pan, Asymptotic incidence energy of lattices, Physica A. 422 (2015) 193-202.

[22] J.B. Liu, J. Cao, A. Alofi, A. AL-Mazrooei, A. Elaiw, Applications of Laplacian spectra for n-Prism networks, Neurocomputing, in press.

[23] J.B. Liu, X.F. Pan, F.T. Hu, F.F. Hu, Asymptotic Laplacian-energy-like invariant of lattices, Appl. Math. Comput. 253 (2015) $205-214$.

[24] J.B. Liu, J. Cao, The resistance distances of electrical networks based on Laplacian generalized inverse, Neurocomputing. 167 (2015) 306313.

[25] J.B. Liu, X.F. Pan, A unified approach to the asymptotic topological indices of various lattices, Appl. Math. Comput. 270 (2015) 62-73.

[26] C. McLeman, E. McNicholas, Spectra of coronae, Linear Algebra Appl. 435 (2011) 998-1007.

[27] S. Y. Cui, G. X. Tian, The spectrum and the signless Laplacian spectrum of coronae, Linear Algebra Appl. 437 (2012) $1692-1703$.

[28] S. Barik, S. Pati, B.K. Sarma, The spectrum of the corona of two graphs, SIAM J. Discrete Math. 24 (2007) $47-56$.

[29] F. Z. Zhang, The Schur Complement and its Applications, Springer, 2005.

[30] I. Gutman, B. Mohar, The quasi-Wiener and the Kirchhoff indices coincide, J. Chem. Inf. Comput. Sci. 36 (1996) $982-985$

[31] H. Y. Zhu, D.J. Klein, I. Lukovits, Extensions of the Wiener number, J. Chem. Inf. Comput. Sci. 36 (1996) $420-428$.

[32] W. Wang, D. Yang, Y. Luo, The Laplacian polynomial and Kirchhoff index of graphs derived from regular graphs, Discrete Appl. Math. 161 (2013) 3063-3071.

[33] B. Mohar, The Laplacian spectrum of graphs, Graph theory, Combinatorics and Applications. 2(1991) 871-898.

[34] F.R.K.Chung, Spectral Graph Theory, CBMS, 1997.

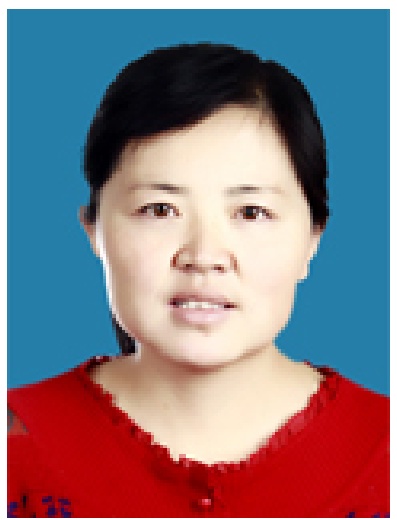

Qun Liu received the B.S. degree in Mathematics education from Northwest Normal University, Lanzhou, China, in 2003, and the M.S. degree in fundamental mathematics from Lanzhou University, Lanzhou, China, in 2009, respectively. She is currently a lecturer, and is working toward the Ph.D. degree at Lanzhou University, Lanzhou, China. 
Her current research interests include graph theory and its applications and complex dynamical networks.

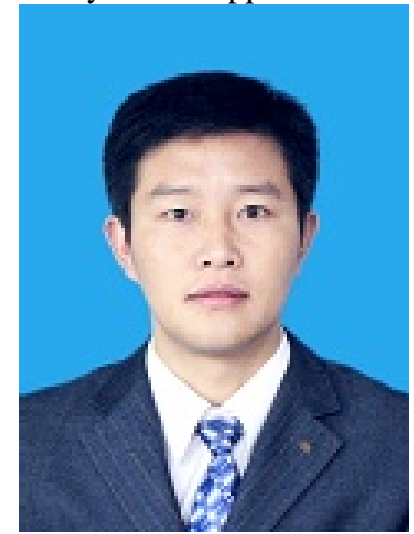

Jia-Bao Liu received the B.S. degree in mathematics and applied mathematics from Wanxi University, Anhui, China, in 2005, and the M.S. degree in mathematics and applied mathematics from Anhui University, Anhui, China, 2009, respectively. He was a visiting researcher with Southeast University, Nanjing, China, in 2013. He is currently a associate professor, and is working toward the PH.D. degree at Anhui University, Hefei, China. His current research interests include fractional calculus theory, graph theory and its applications, neural networks, and complex dynamical networks.

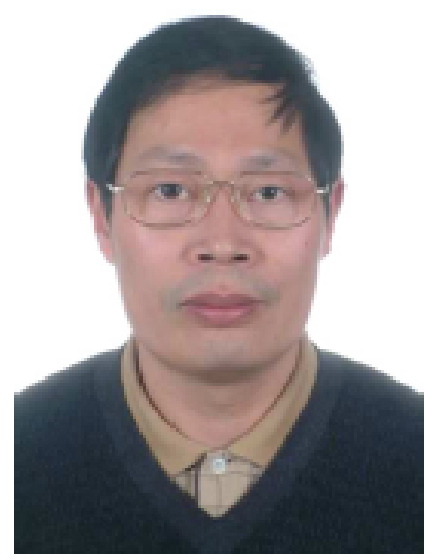

Jinde Cao (M'07-SM'07) Jinde Cao received the B.S. degree from Anhui Normal University, Wuhu, China, the M.S. degree from Yunnan University, Kunming, China, and the Ph.D. degree from Sichuan University, Chengdu, China, all in mathematics/applied mathematics, in 1986, 1989 and 1998, respectively. From March 1989 to May 2000, he was with the Yunnan University. In May 2000, he joined the Department of Mathematics, Southeast University, Nanjing, China. From July 2001 to June 2002, he was a Postdoctoral Research Fellow at the Department of Automation and Computer-Aided Engineering, Chinese University of Hong Kong, Hong Kong. In the period from 2006 to 2008, he was a Visiting Research Fellow and a Visiting Professor at the School of Information Systems, Computing and Mathematics, Brunel University, UK. On August 2014, he was a Visiting Professor at the School of Electrical and Computer Engineering, RMIT University, Australia. Currently, he is a distinguished professor and doctoral advisor at the Southeast University and also distinguished adjunct professor at the King Abdulaziz University, prior to which he was a Professor at Yunnan University from 1996 to 2000. He is the author or coauthor of more than 300 journal papers and five edited books. His research interests include nonlinear systems, neural networks, complex systems and complex networks, stability theory, and applied mathematics. Dr. Cao was an Associate Editor of the IEEE Transactions on Neural Networks, Journal of the Franklin Institute and Neurocomputing. He is an Associate Editor of the IEEE Transactions on Cybernetics, Differential Equations and Dynamical Systems, Mathematics and Computers in Simulation, and Neural Networks. Dr. Cao is a Reviewer of Mathematical Reviews and Zentralblatt-Math. He is a ISI Highly-Cited Researcher in Mathematics and Engineering listed by Thomson Reuters. 
Qun Liu received the B.S. degree in Mathematics education from Northwest Normal University, Lanzhou, China, in 2003, and the M.S. degree in fundamental mathematics from Lanzhou University, Lanzhou, China, in 2009, respectively. She is currently a lecturer, and is working toward the Ph.D. degree at Lanzhou University, Lanzhou, China. Her current research interests include graph theory and its applications and complex dynamical networks.

Jia-Bao Liu received the B.S. degree in mathematics and applied mathematics from Wanxi University, Anhui, China, in 2005, and the M.S. degree in mathematics and applied mathematics from Anhui University, Anhui, China,

2009, respectively. He was a visiting researcher with Southeast University, Nanjing, China, in 2013. He is currently a associate professor, and is working toward the PH.D. degree at Anhui University, Hefei, China. His current research interests include fractional calculus theory, graph theory and its applications, neural networks, and complex dynamical networks.

Jinde Cao received the B.S. degree from Anhui Normal University, Wuhu, China, the M.S. degree from Yunnan University, Kunming, China, and the Ph.D. degree from Sichuan University, Chengdu, China, all in mathematics/applied mathematics, in 1986, 1989 and 1998, respectively. From March 1989 to May 2000, he was with the Yunnan University. In May 2000, he joined the Department of Mathematics, Southeast University, Nanjing, China. From July 2001 to June 2002, he was a Postdoctoral Research Fellow at the Department of Automation and Computer-Aided Engineering, Chinese University of Hong Kong, Hong Kong. In the period from 2006 to 2008, he was a Visiting Research Fellow and a Visiting Professor at the School of Information Systems, Computing and Mathematics, Brunel University, UK. On August 2014, he was a Visiting Professor at the School of Electrical and Computer Engineering, RMIT University, Australia. Currently, he is a distinguished professor and doctoral advisor at the Southeast University and also distinguished adjunct professor at the King Abdulaziz University, prior to which he was a Professor at Yunnan University from 1996 to 2000. He is the author or coauthor of more than 300 journal papers and five edited books. His research interests include nonlinear systems, neural networks, complex systems and complex networks, stability theory, and applied mathematics. Dr. Cao was an Associate Editor of the IEEE Transactions on Neural Networks, Journal of the Franklin Institute and Neurocomputing. He is an Associate Editor of the IEEE Transactions on Cybernetics, Differential Equations and Dynamical Systems, Mathematics and Computers in Simulation, and Neural Networks. Dr. Cao is a Reviewer of Mathematical Reviews and Zentralblatt-Math. He is a ISI Highly-Cited Researcher in Mathematics, Computer Science and Engineering (Thomson Reuters). 